\title{
MORPHOLOGICAL QUANTIFICATION OF AORTIC CALCIFICATION FROM LOW MAGNIFICATION IMAGES
}

\author{
Jesús Angulo ${ }^{1}$, Thao Nguyen-KhoA ${ }^{2}$, Ziad A. Massy ${ }^{2}$, Tilman DrüEKe ${ }^{2}$ And \\ JEAN SERRA ${ }^{1}$ \\ ${ }^{1}$ Centre de Morphologie Mathématique, Ecole des Mines de Paris, 35, rue Saint-Honoré, 77305 Fontainebleau, \\ France, ${ }^{2}$ Laboratoire INSERM U 507, Hôpital Necker, 161, rue de Sèvres, 75015 Paris, France \\ e-mail: \{angulo,serra\}@cmm.ensmp.fr,massy@necker.fr \\ (Accepted May 14, 2003)
}

\begin{abstract}
Atherosclerotic and medial vascular calcifications are frequent in chronic renal failure patiens and predict their increased cardiovascular mortality. Experimental models for mice have been recently developed in order to study these disorders. The aim of this paper is to present the morphological image processing algorithms developed for the semi-automated measurement of calcification from sections of aorta stained using von Kossa's silver nitrate procedure and acquired at low magnification power $(\times 2.5)$ on colour images. The approach is separated into two sequential phases. First, the segmentation is aimed to extract the calcification structures and on the other hand to demarcate the region of the atherosclerotic lesion within the tissue. The segmentation yields the image data which is the input to the second phase, the quantification. Calcified structures are measured inside and outside the lesion using a granulometric curve which allows the calculation of statistical parameters of size. The same operator computes the shape of the lesion. The relative proportion of the area of calcification is also calculated respectively for the atherosclerotic lesion area and the area outside such lesions. In conclusion, the here developed method allows quantification of vascular calcified deposits in mouse aorta. This method will be useful for the quantitative assessment of pathological vascular changes in animals and man.
\end{abstract}

Keywords: aortic calcification, automation in bioimaging, low magnification histology, mathematical morphology, nephrology, quantitative image analysis.

\section{INTRODUCTION}

Atherosclerotic and medial vascular calcifi cations are frequent in chronic renal failure patients and predict their increased cardiovascular mortality. Experimental models for mice have been proposed in order to study these disorders (Muntzel et al., 2002). The detection of coronary artery calcium, using mainly fluoroscopy, has been shown to be of value in predicting artery disease (Margolis et al., 1980; Reinmuller and Lipton, 1987; Tanenbaum et al., 1989). Recently, electron beam tomography has been introduced for the detection of coronary calcium (Agatston et al., 1990; Morgan-Hughes et al., 2002).

The purpose of the present work is to propose an image-based method of quantifi cation for the uremiaenhanced vascular calcification in the aortic root, which allows the evaluation of previously established biological models.

The images were derived from thin sections of aortic tissue. The aim is the extraction and quantifi cation of calcification deposits. Confi rmation of the presence of calcifi cation is provided by staining using von Kossa's silver nitrate method which is widely used for detection of calcifi cation (Lardenoye et al., 2002).

In previous studies, mathematical morphology has shown its ability for solving bioimaging problems from low magnification power images in haematological cytology (Angulo and Flandrin, 2003). Such morphological approaches have also been applied successfully to other issues in nephrology (Moreso et al., 1994; Seron et al., 1996).

\section{MATERIALS AND METHODS}

\section{STAINED AORTIC LOW MAGNIFICATION IMAGE}

The model of apolipoprotein $\mathrm{E}$ gene knockout mice has been initially created by homologous recombination in embryonic stem cells. This genetically engineered mouse generates atherosclerotic lesions within weeks after birth that are similar to those found in humans (Zhang et al., 1992; Ishibashi et al., 1994). We created chronic renal failure in these mice by cortical electrocauterization in one kidney and ablation of the contralateral kidney 
two weeks later. After sacrifi ce, the aortic arch was cut into serial sections in a cryostat. Sections were stained with von Kossa's silver nitrate method. The animals were treated according to the recommendations of animal care committees, under anesthesia (Protocol: short term anesthesia of a duration of 30-40 min; one IP injection composed of $75 \mu$ Rompun $2 \%$ in $25 \mathrm{ml}$ vial, $300 \mu$ Ketamine CLORKETAM 1000 in $10 \mathrm{ml}$ vial, $1.53 \mathrm{ml} 0.09 \% \mathrm{NaCl}$; the amount of the anesthetic fluid administered $100-120 \mu \mathrm{l} / 20 \mathrm{~g}$ body weight).

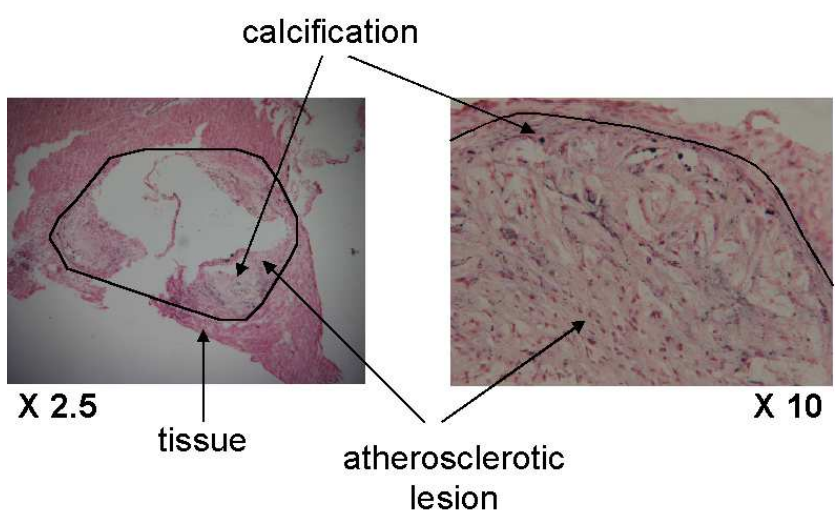

Figure 1. Images under two magnification powers $(\times 2.5$ and $\times 10)$ of $a$ section of the aortic sinus showing the elements of interest: tissue, atherosclerotic lesion and calcification structures.

In Fig. 1 are depicted two colour images under two magnification powers $(\times 2.5$ and $\times 10)$ of a section of the aortic sinus showing the elements of interest: tissue, atherosclerotic lesion and calcifi cation structures. In this study, the series of colour images were acquired under very low magnifi cation power at $\times 2.5$. The main advantage is that in one image fi eld all the tissue to be examined is included; however there is a considerable drawback since the quality of the structures is mediocre. Therefore the automation of the segmentation and quantific cation procedures involves the development of specifi $\mathrm{c}$ algorithms using advanced techniques of image analysis. This is the rationale behind the use of mathematical morphology operators. As one can see from Fig. 1, other alternatives are possible. For instance, working at the magnification power of $\times 10$ and using a motorized microscope is an alternative to acquire several subimages to cover the whole area of interest. This approach has however serious drawbacks including defi nition of lesion at $\times 10$, extraction of the whole calcification region and merger of quantifi ed data (overlapping of images).

The size of the image is $672 \times 538$ pixels on a rectangular raster with 256 grey tone levels for each red, green and blue colour channels. We consider for quantification that at $\times 2.5$ magnifi cation and for the resolution used, the size of a pixel is $5.5 \mu \mathrm{m}^{2} /$ pixel.
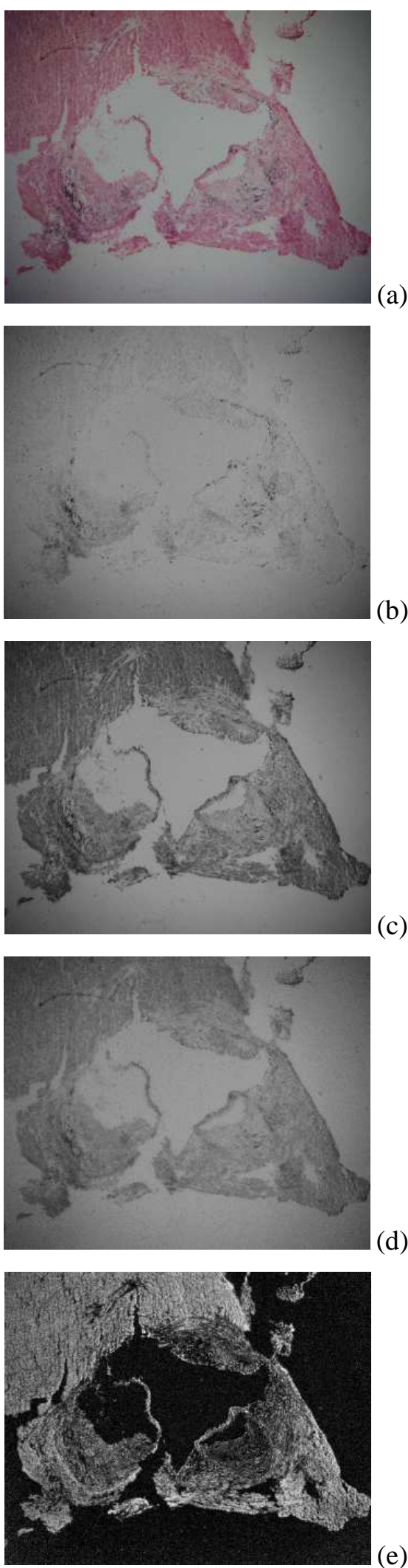

Figure 2. Example of microscopic field image from a section of aorta stained using the von Kossa method at low magnification power $(\times 2.5)$ : (a) RGB colour image $\mathbf{f}_{R G B},(b)$ red component $f_{R}$, (c) green component $f_{G}$, (d) blue component $f_{B}$, (e) saturation component $f_{S}$. 
Fig. 2 is an example of colour image to be processed, $\mathbf{f}_{R G B}$. The red $f_{R}$, green $f_{G}$ and blue $f_{B}$ colour component images are shown and beside this, the saturation component $f_{S}$ of a 3D-polar coordinate colour representation (Hanbury and Serra, 2002). For a colour pixel $\mathbf{c}=(r, g, b)$; i.e. $\mathbf{f}_{R G B}(\mathbf{c})=$ $\left(f_{R}(\mathbf{c}), f_{G}(\mathbf{c}), f_{B}(\mathbf{c})\right)$ the saturation coordinate $f_{S}(\mathbf{c})$ is obtained by the simple expression

$$
f_{S}(\mathbf{c})=\max (r, g, b)-\min (r, g, b) .
$$

Using the spectral properties of the staining procedure on the tissues (calcifi cations appear black and tissues red-pink), we use the colour component images in the following way:

- The calcification is relatively more contrasted against the rest of tissues in $f_{R}$, therefore this component is used for extracting the calcifi cations.

- In order to detect the tissue in the fi eld image the simplest means is to take $f_{S}$ where the biological structures are clearly defi ned, despite the shading effect (non-uniform illumination).

- For segmenting the region of the atherosclerotic lesion a combined method of thresholding and manual interaction is achieved from $f_{G}$.

Through this choice of the colour components, the color shading effect is negligible. Anyway, a method for shading correction of color images (Tomaževič et al., 2002; Derganc et al., 2003) can be used in applications with important multispectral inhomogeneities.

\section{MORPHOLOGICAL IMAGE PROCESSING AND QUANTIFICATION}

First introduced as a shape-based tool for binary images, mathematical morphology has become a very powerful nonlinear image analysis technique with operators capable of handling sophisticated image processing tasks in binary, grey-scale, colour and multiresolution imaging modalities. A tutorial in the technique can be found in (Serra, 1982, 1988; Coster and Chermant, 1989). In this section we briefly review the basic morphological operators used in this work.

In the framework of digital grids, a grey-tone image can be represented by a function $f: D_{f} \rightarrow T$, where $D_{f}$ is a subset of $\mathbf{Z}^{2}$ and $T=\left\{t_{\min }, \ldots, t_{\max }\right\}$ is an ordered set of grey-levels. Let $B$ be a subset of $\mathbf{Z}^{2}$ and $s \in \mathbf{N}$ a scaling factor. $s B$ is called structuring element (shape probe) $B$ of size $s$. The basic morphological operators are

- Dilation: $\delta_{B}(f(x))=\sup _{y \in B}\{f(x-y)\}$

\section{- Erosion: $\varepsilon_{B}(f(x))=\inf _{-y \in B}\{f(x-y)\}$}

The two elementary operations of erosion and dilation can be composed together to yield a new set of operators having desirable feature extractor properties which are given by

- Opening: $\gamma_{B}(f)=\delta_{B}\left[\varepsilon_{B}(f)\right]$

- Closing: $\varphi_{B}(f)=\varepsilon_{B}\left[\delta_{B}(f)\right]$

The morphological openings (closings) fi lter out light (dark) structures from the images according to the predefi ned size and shape criterion of the structuring element.

The top-hat transformation is a powerful operator which permits the detection of contrasted objects on non-uniform backgrounds (Meyer, 1977). There are two versions,

- White top-hat: The residue of the initial image $f$ and an opening $\gamma(f)$; i.e. $\rho(f)=f-\gamma(f)$, extracts bright structures.

- Black top-hat: The residue of a closing $\varphi(f)$ and the initial image $f ; \rho^{*}(f)=\varphi(f)-f$, extracts dark structures.

Usually, the top-hat is accompanied by a thesholding operation, in order to binarise the extracted structures.

A granulometry is the study of the size distributions of the objects of an image. Formally, a granulometry can be defi ned as a family of openings $\Gamma=\left(\gamma_{n}\right)_{n \geq 0}$ such that $\forall n \geq 0, \forall m \geq 0, \gamma_{n} \gamma_{m}=\gamma_{m} \gamma_{n}=$ $\gamma_{\max (n, m)}$. Moreover, granulometries by closings (or anti-granulometry) can also be defi ned as families of increasing closings $\Phi=\left(\varphi_{n}\right)_{n \geq 0}$. Performing the granulometric analysis of an image $f$ with $\Gamma$ is equivalent to mapping each opening of size $n$ with a measure $\mathscr{M}\left(\gamma_{n}(f)\right)$ of the opened image. $\mathscr{M}(f)$ is the area of $f$ in the binary case (number of pixels) and the volume in the grey scale case (sum of pixel values). The size distribution or pattern spectrum of $f$ with respect to $\Gamma$, denoted $P S_{\Gamma}(f)$ is defi ned as the following (normalised) mapping

$$
\begin{aligned}
P S_{\Gamma}(f, n) & =P S(f, n)= \\
& \frac{\mathscr{M}\left(\gamma_{n}(f)\right)-\mathscr{M}\left(\gamma_{n+1}(f)\right)}{m(f)}, \quad n \geq 0 .
\end{aligned}
$$

The pattern spectrum $P S_{\Gamma}(f)$ maps each size $n$ to some measure of the bright image structures with this size (loss of bright image structures between two successive openings). The pattern spectrum $P S_{\Gamma}(f, n)$ is a probability density function (a histogram): a large impulse in the pattern spectrum at a given scale indicates the presence of many image structures at that 
scale. It is also possible to use standard probabilistic defi nitions to compute the moments of $P S$. The fi rst moment $\mu$ is given by $\mu=\mu^{1}=\sum_{n} n P S(f, n)$, the $k$-th pattern spectrum moment, $k \geq 2$, is computed as $\mu^{k}=\sum_{n}(n-\mu)^{k} P S(f, n)$. In particular, the fi rst four moments: mean $\mu$, variance $\mu^{2}$, skewness $\mu^{3}$ and kurtosis $\mu^{4}$ are often used.

Using a pair of an opening $\gamma$ and a closing $\varphi$ as primitives, an operator of contrast enhancement can be obtained (Serra, 1989). This toggle mapping or two states contrast $\kappa$ is generated by the following criterion applied to each point $x$,

$$
\kappa(f(x))=\left\{\begin{array}{c}
\gamma(f(x)) \text { if } \\
\varphi(f(x))-f(x)>f(x)-\gamma(f(x)) \\
\varphi(f(x)) \text { if } \\
\varphi(f(x))-f(x)<f(x)-\gamma(f(x)) \\
f(x) \text { if } \\
\varphi(f(x))-f(x)=f(x)-\gamma(f(x))
\end{array}\right.
$$

The closing and the opening may be replaced by a dilation and an erosion of $f$.

A morphological tool that complements the opening and closing operators for feature extraction (extract the marked particles) is the morphological reconstruction, implemented using the geodesic dilation operator based on restricting the iterative dilation of a function marker $f$ by $B$ to a function mask $g, \delta_{g}^{n}(f)=\delta_{g}^{1} \delta_{g}^{n-1}(f)$, where $\delta_{g}^{1}(f)=\delta_{B}(f) \wedge g$. The reconstruction by dilation is defi ned by

$$
\gamma^{r e c}(g, f)=\delta_{g}^{i}(f)
$$

such that $\delta_{g}^{i}(f)=\delta_{g}^{i+1}(f)$ (idempotence).

The thresholding transformation of the image $f$ between the grey levels $u_{1}$ and $u_{2}$ (typically $u_{1}=t_{\text {min }}$ or $\left.u_{2}=t_{\max }\right), T_{\left[u_{1}, u_{2}\right]}(f)$, yields the binary image $I$ such that for each pixel $x$ the binary value is given by

$$
I(x)=T_{\left[u_{1}, u_{2}\right]}(f(x))= \begin{cases}1 & u_{1} \leq f(x) \leq u_{2} \\ 0 & \text { otherwise }\end{cases}
$$

The choice of $\left[u_{1}, u_{2}\right]$ determines the set of grey levels associated with the object of interest. The histogram summarises the grey-level contents of an image and typically, the optimal threshold values can be obtained from an analysis of the histogram, see in (Angulo and Flandrin, 2003) the method of automated thresholding which combines the classical selection of the threshold value by minimising the sum of within class variances with a morphological technique for selecting the central mode values which speeds up the convergence to the $u$ 's. An alternative to make the choice of $\left[u_{1}, u_{2}\right]$ easier is a method which relies on a double thresholding combined with a geodesic reconstruction; the technique is known as thresholding by hysteresis (Soille, 1999). For instance, in order to extract light structures we have to take $u_{2}=t_{\text {max }}$ and let $u_{T_{l o w}}$ be a low threshold value and $u_{T_{\text {high }}}$ be a high threshold value. Using this double threshold, two

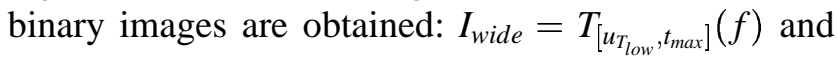
$I_{\text {narrow }}=T_{\left[u_{T_{\text {high }},}, t_{\text {max }}\right]}(f)$. The fi nal binary image is given by the reconstruction of $I_{\text {wide }}$ using $I_{\text {narrow }}$ as a marker; i.e.

$$
I=T_{\left[u_{T_{l o w}}<>u_{T_{\text {high }}}, t_{\max }\right]}^{\text {hyt }}(f)=\gamma^{\text {rec }}\left(I_{\text {narrow }}, I_{\text {wide }}\right) .
$$

\section{ALGORITHMS}

The approach is separated into two sequential phases. First, the segmentation is aimed on the one hand to extract the calcium structures and on the other hand to demarcate the region of the lesion on the tissue. The segmentation yields the image data which are the input to the second phase, the quantifi cation. The calcifi cation structures are measured inside and outside the lesion using a granulometric curve which allows to calculate some statistical parameters of size. The same operator is used to compute the shape of the lesion. The relative proportion of area of calcifi cation is also calculated.

\section{Detection of tissue in the field image}

We start by binarising the saturation component $f_{S}$ for detecting the tissue presented in the field image by using a simple thresholding operation at $u_{T_{1}}$ (a low value; e.g. $u_{T_{1}}=10$, has shown to be suitable for this kind of images), see Fig. 3(a),

$$
I_{s}=T_{\left[u_{T_{1}}, t_{\text {max }}\right]}\left(f_{S}\right) .
$$

In order to remove the noise and mistakes due to the small pieces of tissue (histology artefact), the image is then filtered by applying a reconstruction using an opening as the marker,

$$
I_{\text {tissue }}=\gamma^{\text {rec }}\left(\gamma_{s_{1} B}\left(I_{S}\right), I_{S}\right),
$$

with $s_{1}$ such that $s_{1} B$ is larger than the size of the noise and the small artefact pieces of tissue (good results were obtained with $s_{1}=5$ ); the structuring element $B$ is a circle. See the result in Fig. 3(b). 

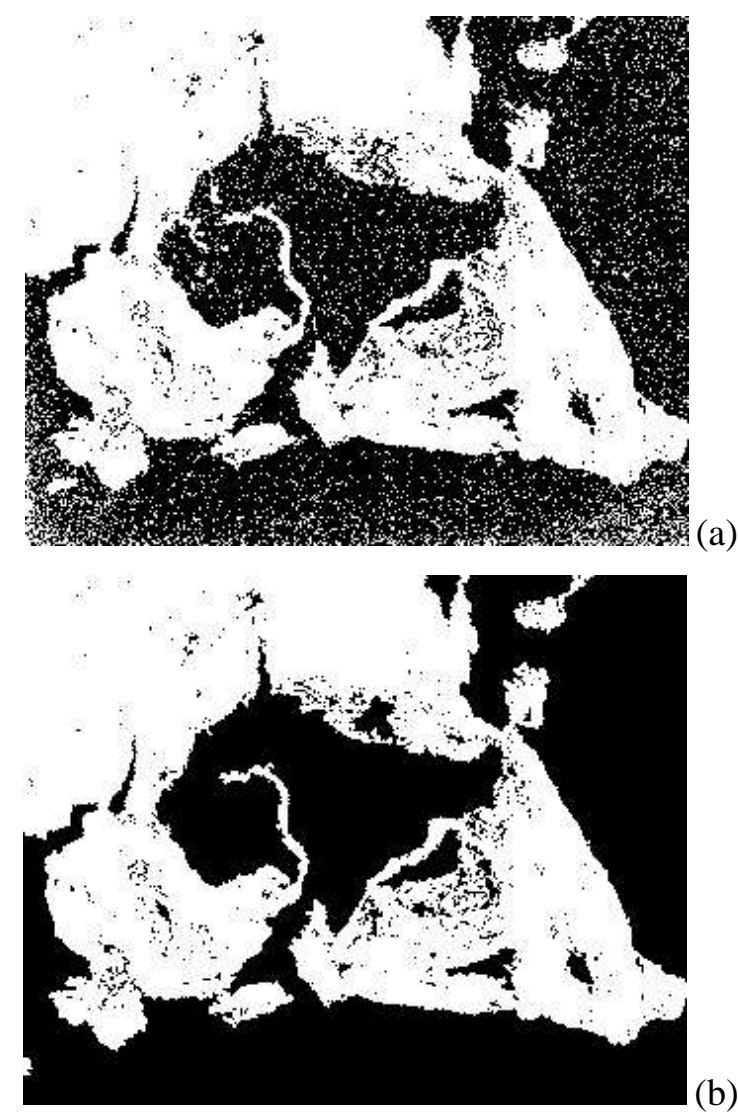

(b)

Figure 3. Detection of tissue in the field image: (a) Binary image after thresholding saturation component, $I_{s}$; (b) cleaned binary mask of tissue, $I_{\text {tissue }}$.

\section{Interactive segmentation of the region of atherosclerotic lesion}

In the tissue, the atherosclerotic lesion zone squares with a texture of tissue less thick than the rest, recognising visually, but its precise morphological defi nition is almost impossible. Therefore due to the fact that the automated segmentation of the lesion on the tissue can not be achieved without some mistakes and in order to avoid the propagation of these errors to the quantifi cation step, we preferred to developed an interactive approach.

In a fi rst step the human expert must perform a manual dot-marking of the region of interest (ROI) using the computer mouse on the colour image. This zone associated to the lesion has to be closed, see Fig. 4(d), but it is possible for the human user to demarcate several closed partial ROI's: the defi nitive ROI is the union of the partial ones and is represented by the binary image $I_{m r k}$.

The automated step works on the green component $f_{G}$. This image is simplifi ed by means of an opening by reconstruction (which simplifi es the light structures),

$$
f_{G}^{\prime}=\gamma^{r e c}\left(\gamma_{s_{2} B}\left(f_{G}\right), f_{G}\right)
$$
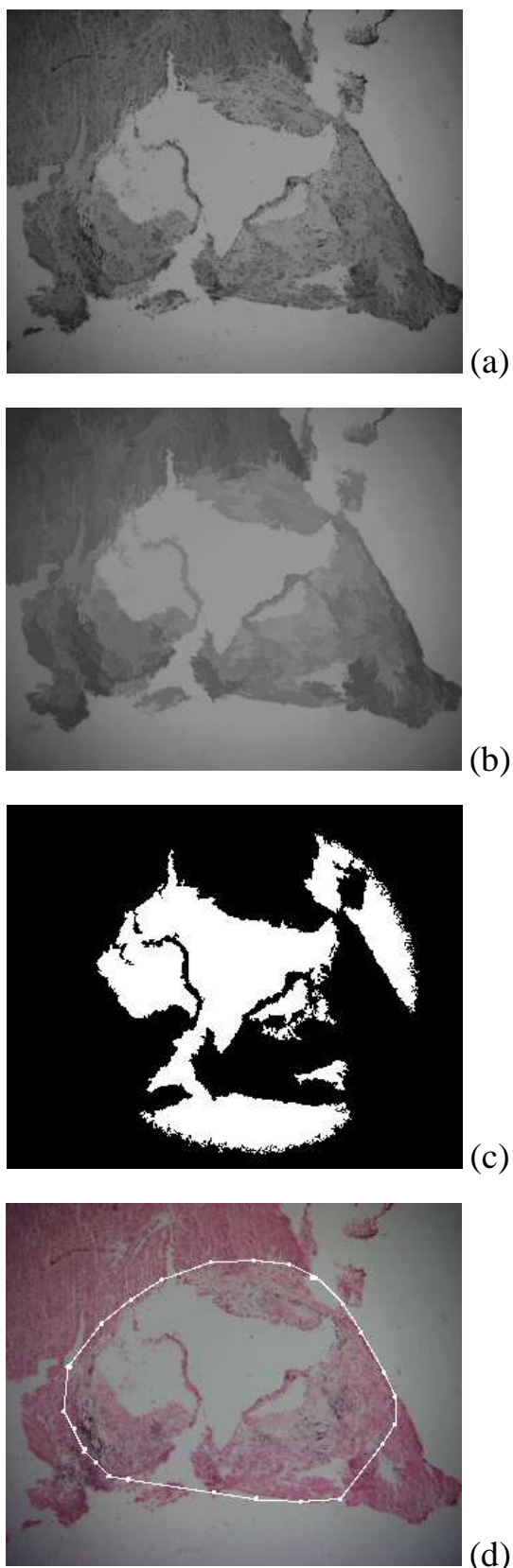

c)

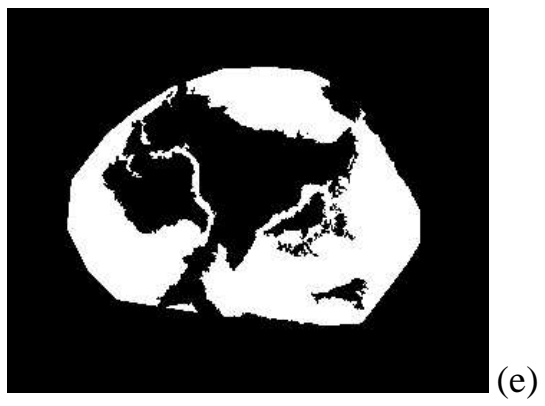

Figure 4. Interactive segmentation of the region of lesion: (a) Simplified green component using an opening by reconstruction, $f_{G}^{\prime}$; (b) followed by closing by reconstruction, $f_{G}^{\prime \prime}$; (c) negative of the binary mask without empty image zones, $\bar{I}_{g}$; (d) manual dotmarking region of interest, $I_{m r k}$; (e) binary mask of lesion inside the region of interest, $I_{\text {lesion }}$. 
followed by a closing by reconstruction (which simplifi es the dark structures),

$$
f_{G}^{\prime \prime}=\varphi^{r e c}\left(\varphi_{s_{3} B}\left(f_{G}^{\prime}\right), f_{G}^{\prime}\right),
$$

where the sizes of the opening and the closing have been empirically fixed to $s_{2}=15$ and $s_{3}=$ 10 respectively ( $B$ is a circle). The corresponding example of tissue image after simplification is shown in Fig. 4(a)-(b). The image $f_{G}^{\prime \prime}$ must be binarised for extracting the empty zones (zones of the fi eld without tissue); the optimal threshold value $u_{T_{2}}$ for each image is obtained by the algorithm presented in Angulo (02). The result of this thresholding process at $u_{T_{2}}$,

$$
I_{g}=T_{\left[t_{\min }, u_{T_{2}}\right]}\left(f_{G}^{\prime \prime}\right),
$$

is a fi rst binary mask which is then restricted to the manual defi ned $I_{n r k}$ to obtain the binary mask of the region of lesion,

$$
I_{\text {lesion }}=I_{m r k} \wedge I_{g}
$$

See the result in Fig. 4(e).

\section{Extraction of calcification}

In the red component of the aortic section colour image $f_{R}$ the calcium appears as dark structures upon a bright background. In order to enhance the contrast of the calcifi cations against the background a toggle mapping is taken,

$$
\widehat{f}_{R}=\kappa\left(f_{R}\right),
$$

where the two primitives of $\kappa$ are an erosion and a dilation of size 3, see Fig. 5(a). From this image, a black top-hat of size $s_{4}$ extracts the calcification ( $s_{4}$ corresponds to the size of the biggest calcium structures which can be found); experimentally we have fi xed $s_{4}=25$ with a circular structuring element,

$$
f_{c}=\rho^{*}\left(\widehat{f}_{R}\right) .
$$

On the image $f_{c}$, Fig. 5(b), a thresholding by hysteresis is performed to provided the binary mask of calcifi cations (see Fig. 5(c)),

$$
I_{\text {calcif }}=T_{\left[u_{T_{l o w}}^{\text {hst }}<>u_{\left.T_{\text {high }}, t_{\text {max }}\right]}(f)\right.}(f
$$

the choice of the threshold values is not so critical (the top-hat facilitates just the thresholding); e.g. $u_{T_{\text {low }}}=50$ and $u_{T_{h i g h}}=70$.
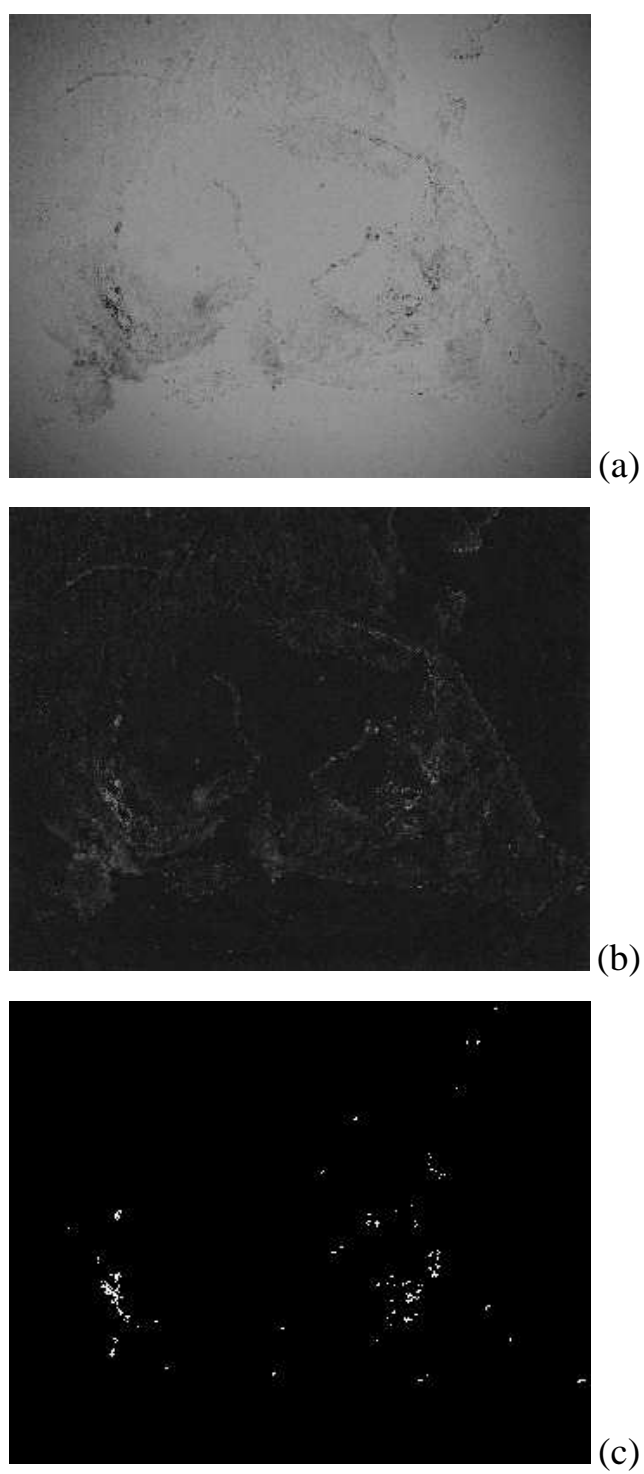

Figure 5. Extraction of calcification: (a) Contrast enhancement of calcification on red component, $\widehat{f}_{R} ;(b)$ extracted calcification by means of a dual top-hat, $f_{c}$; (c) binary mask of calcification after thresholding by hysteresis, $I_{\text {calcif }}$.

\section{Quantification of atherosclerotic lesion}

Let $\mathscr{A}(I)$ be the surface area; i.e. number of pixel to ones, of the binary image $I$. We start by calculating the size of the tissue and the lesion regions,

$$
A_{\text {tissue }}=\mathscr{A}\left(I_{\text {tissue }}\right), \quad A_{\text {lesion }}=\mathscr{A}\left(I_{\text {lesion }}\right) .
$$

We propose to characterise the shape of the lesion region by means of a pattern spectrum curve, $P S\left(I_{\text {lesion }}, n\right)=P S_{\text {lesion }}$. The structuring elements are circles of increasing size $n+2$ (in fact, the practical shape is an octagon: isotropic approach to a circle in the square grid), from $n=4$ to $n=70$. The granulometric curves describe in a compact way the different thickness of the wall of the aortic valves: each 
peak corresponds to the thickness of a sector of the wall. These histograms of shape can be parametrised by using their statistical moments. The surface area in pixels of an octagon of size $n$ is given by the formula (Serra, 1982), $N_{\text {octagon }}=7 n^{2}+4 n+1$. In order to obtain the sizes in $\mu \mathrm{m}^{2}$ we have to multiply the area in pixels by $5.5 \mu \mathrm{m}^{2} /$ pixel for all the measurements.

\section{Quantification of calcification}

In order to quantify the calcium, we compute fi rst the absolute and the relative surface of calcifi cations inside and outside the lesion,

$$
\begin{aligned}
& A_{\text {calcif } \_i_{i}}=\mathscr{A}\left(I_{\text {calcif }} \wedge I_{\text {lesion }}\right), \\
& A_{\text {calcif॰o }}=\mathscr{A}\left(I_{\text {calcif }} \wedge\left(I_{\text {tissue }}-I_{\text {lesion }}\right)\right) \text {, } \\
& \bar{A}_{\text {calcif } \_i}=\frac{A_{\text {calcif_i } i}}{\mathscr{A}\left(I_{\text {lesion }}\right)}, \\
& \bar{A}_{\text {calcif_o }}=\frac{A_{\text {calcif_o }}}{\mathscr{A}\left(I_{\text {tissue }}-I_{\text {lesion }}\right)} .
\end{aligned}
$$

Besides these parameters, the calcifi cation structures inside the lesion are quantified by using a size distribution, $P S\left(I_{\text {calcif }} \wedge I_{\text {lesion }}, n\right)=P S_{\text {calcif } j}$ (the structuring elements of openings are again circles of increasing size $n+2$, from $n=3$ to $n=23$ ) and its moments.

\section{RESULTS AND DISCUSSION}

In Fig. 6 two examples of segmented aortic section images are depicted: one corresponding to a mouse with low calcifi cation content (control mouse) and another to high calcifi cation (uremic mouse). In Fig. 7 their associated pattern spectra are shown.

In Table 1 the obtained parameters of the lesion are included. For mouse $\sharp 35$ the area of lesion is larger than for mouse $\sharp 40$, but above all, the thickness of the walls is considerably greater. Besides being used for determining the relative amount of calcium located inside, the size of the atherosclerotic lesion may be signifi cant a priori. However, due to the fact that during the tissue preparation procedure the shape of the atherosclerotic lesion may be modifi ed involuntarily, the practical usefulness of the parameters from $P S_{\text {lesion }}$ remains doubtful. Other techniques of tissue preparation could be envisaged in order to achieve more reproducible sections in such a way that the parameters associated to $P S_{\text {lesion }}$ will be very important.
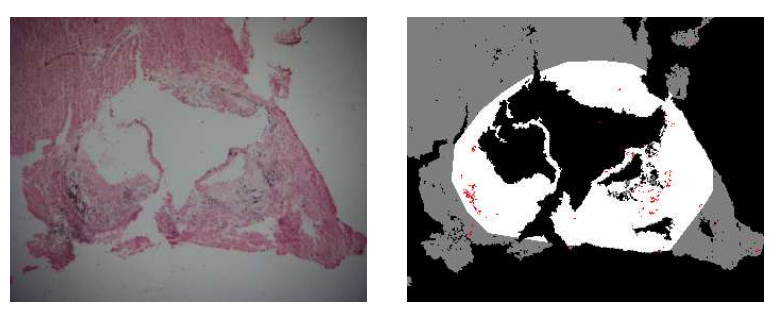

(a)
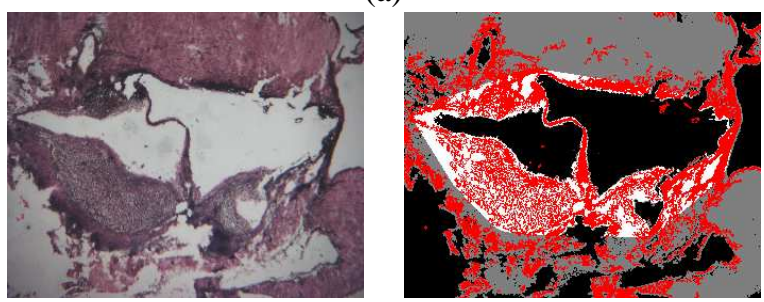

(b)

Figure 6. Two examples of aortic section images: (a) with low calcification content (mouse $\sharp 35$ ); $(b$ ) riches in calcification (mouse $\sharp 40$ ). On the left, the initial images and on the right, summary of segmented structures: the background in black, the tissue in gray, the lesion in white and the calcification in red.
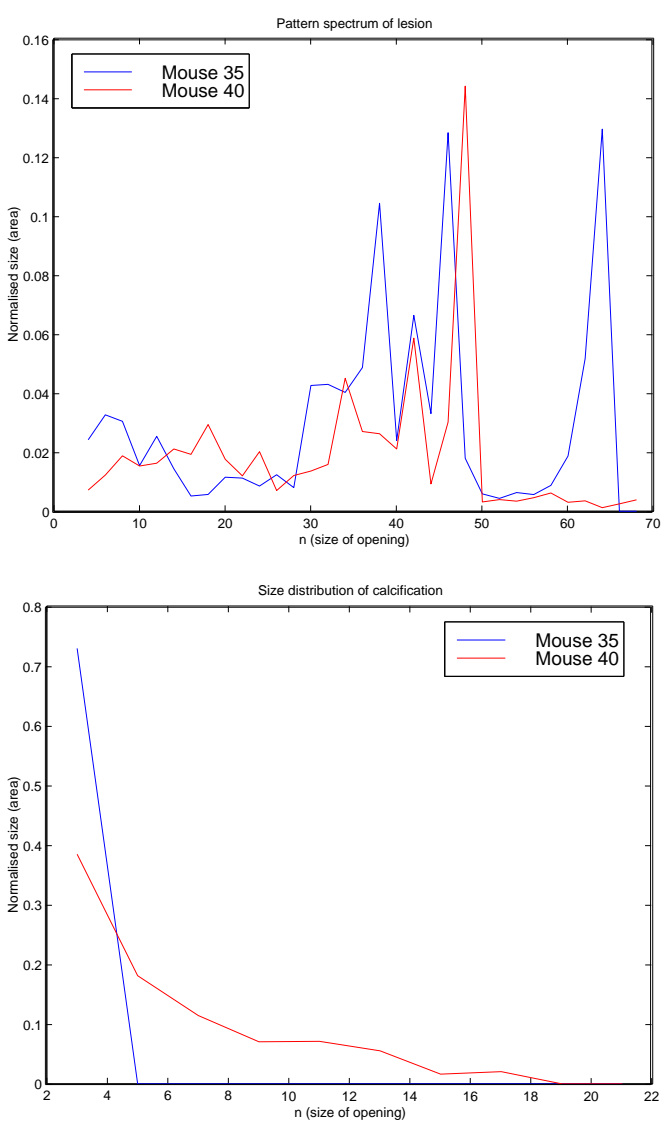

Figure 7. Quantification of size and shape using morphological granulometries (curves associated to the examples of Fig. 6): (a) Pattern spectrum of the region of lesion; (b) Size distribution of calcification structures inside the lesion. 
Table 1. Size $\left(A_{\text {tissue }}\right.$ and $\left.A_{\text {lesion }}\right)$ and shape (first four moments of $P S_{\text {lesion }}$ ) parameters of lesion zone for the examples of Fig. 6.

\begin{tabular}{lcccccc}
\hline & $A_{\text {tissue }}$ & $A_{\text {lesion }}$ & $\mu$ & $\mu^{2}$ & $\mu^{3}$ & $\mu^{4}$ \\
\hline Mouse $\sharp 35$ & 120859 & 59079 & 38.55 & 293.03 & -1176.89 & 208019.51 \\
Mouse $\sharp 40$ & 160483 & 78696 & 51.17 & 647.21 & -206.51 & 720299.94 \\
\hline
\end{tabular}

Regarding the parameters of calcification, summarised in Table 2, the use of this set of parameters yields an easy way to identify the different levels of calcification. Obviously, the most interesting parameters are the relative surfaces of calcification and the two first moments of size distribution: the mean size of calcium structures and the variance of size (which gives an idea of size dispersion and is helpful for distinguishing large compact calcifi cations from large disintegrated calcifi cations).

Table 2. Surface area parameters and first four moments of $P S_{\text {calcif_i }}$ of lesion zone for the examples of Fig. 6.

\begin{tabular}{lcccc}
\hline & $A_{\text {calcif_i } i}$ & $A_{\text {calcif_o }}$ & $\bar{A}_{\text {calcif_i } i}$ & $\bar{A}_{\text {calcif_o }}$ \\
\hline Mouse $\sharp 35$ & 821 & 170 & 0.0139 & 0.0014 \\
Mouse $\sharp 40$ & 43094 & 37137 & 0.5476 & 0.2314 \\
\hline
\end{tabular}

(a)

\begin{tabular}{lcccc}
\hline & $\mu$ & $\mu^{2}$ & $\mu^{3}$ & $\mu^{4}$ \\
\hline Mouse $\sharp 35$ & 2.19 & 0.48 & 0.39 & 0.32 \\
Mouse $\sharp 40$ & 5.59 & 12.92 & 74.21 & 729.99 \\
\hline
\end{tabular}

(b)

The robustness of an algorithm can be defined with respect to changes in the parameters or to image quality. The present algorithms have seven confi gurable parameters: $u_{T_{1}}$ (threshold value for the tissue determination on $f_{S}$ ), $s_{1}$ (size of the tissue filtering on $f_{5}$ ), $s_{2}$ and $s_{3}$ (size of the tissue simplifi cation on $f_{G}$ ), $s_{4}$ (size of the calcium enhancement on $f_{R}$ ), $u_{T_{\text {low }}}$ and $u_{T_{h i g h}}$ (threshold values for the calcium extraction on $f_{R}$ ). The threshold value $u_{T_{2}}$ is obtained automatically for each image. The values proposed for the other parameters have been set after empirical tests on a random selection of 10 images which covers the different levels of pathology and image quality. Someone who would like to use these methods with a different magnification or a different camera has to start by using a training set of images (representative of its problem) in order to adjust the values of parameters. In any case, some tests of images at $\times 10$ have shown that the approach is quite robust.

Once these parameters were fi xed, a deep study has been performed on the basis of the present methods. A database of 173 images corresponding to 45 mice has been used. The results of the interactive human segmentation of the atherosclerotic lesion and the extraction of calcification have been subjected to evaluation by another human grader in order to fi nd out serious mistakes: only 3 cases among the set of 173 have been rejected and manually corrected (1.7\%). In view of these results we can state that the behaviour of the image analysis algorithms is quite satisfactory.

The approach has however several limitations. On the one hand, in order to minimise the errors of segmentation, an interactive algorithm was developed which involves a necessary human action for each image. Obviously, the task is simple (some "clicks" of mouse) but time consuming. The balance between automation and precision leads sometimes to this kind of approach. On the other hand, working on low magnification microscopic images entails that the image structures can be very small, limiting the resolution of the methods. For instance in Fig. 7 (right), notice that the size distribution of calcium for the control mouse (small calcifi cation structures) is limited to the openings of size $n=3$ and 5. The classical problem of reproducibility of histologic preparations (sectioning, staining, etc.) must be also taken into account in order to prevent artefacts, like dust or others.

\section{CONCLUSION}

The robustness and accuracy of segmentation results allowed us to consider the subsequent quantification as a correct procedure. The morphological parameters obtained have been analysed and correlated to several biomedical parameters (Massy et al., 2003). We also envisage to apply this quantification approach to the study of calcification using non-invasive techniques, for instance by obtaining the morphological information by means of electron beam tomography or other imaging technique. In the previous studies based on computed tomography (Agatston et al., 1990, Morgan-Hughes et al., 2002), the quantification of calcification has not been attempted using morphological techniques.

To summarise the algorithmic discussion above, we conclude that quantitative measurements of 
histologic sections by morphological image analysis software are very useful. They provide a powerful tool for improving and automating experimental studies on structural-functional correlations in tissues with histopathologic changes.

\section{ACKNOWLEDGMENTS}

The authors thanks the comments and suggestions of the anonymous reviewers.

\section{REFERENCES}

Agatston AS, Janowitz WR, Hildner FJ, Zusmer NR, Viamonte M, Detrano R (1990). Quantification of Coronary Artery Calcium Using Ultrafast Computed Tomography. J Am Coll Cardiol 15:827-32.

Angulo J, Flandrin G (2003). Automated detection of working area of peripheral blood smears using mathematical morphology. Anal Cell Pathol, 25:37-49.

Coster M, Chermant JL (1989). Pr'ecis d'analyse d'images, 2nd. ed. Paris: Les Presses du CNRS.

Derganc J, Likar B, Bernard R, Tomaževič D, Pernuš F (2003). Real-time automated visual inspection of color tablets in pharmaceutical blisters. Real-Time Imaging, 9:113-24.

Hanbury A, Serra J (2002). A 3D-polar Coordinate Colour Representation Suitable for Image Analysis. Submitted to Comput Vis Image Und.

Ishibashi S, Herz J, Maeda N, Goldstein JL, Brown MS (1994). The two-receptor model of lipoprotein clearence: Tests of the hypothesis in "knockout" mice lacking the low density lipoprotein receptor, apolipoprotein E, or both proteins. Proc Natl Acad Sci USA, 91:4431-5.

Lardenoye J, de Vreis M, lowik C, Xu C,Dhore C, Cleutjens, J van Hinsberg V, van Bockel J, Quax P (2002). Accelerated atherosclerosis and calcification in vein grafts. Circ Res 91:577-84.

Margolis JR, Chen JTT, Kong Y, Peter H, Behar VS, Kisslo JA (1980). The diagnostic and prognostic significance of coronary artery calcification. Radiology 137:609-16.

Massy Z, Nguyen-Khoa T, Angulo J, Munzel M, Ivanovski O, Szumilak D, Ruellan N, Descamps-Latscha B, Lacour B, Drüeke T (2003) Uremia accelerates calcification in apolipoprotein E knock-out (EKO) mice. To be presented in 17th Congress of the International Society of Nephrology - 40th Congress of the European Renal Association, Berlin, June 8-12.
Meyer F (1977). Constrast features extraction. In: Chermant JL, ed. Quantitative Analysis of microstructures in Materials Science, Biology and Medecine. Stuttgart: Riederer Verlag, 374-80.

Moreso F, Ser'on D, Vitri'a J, Griny'o JM, Colom 'e-Serra FM, Par'es N, Serra J (1994). Quantification of interstitial chronic renal damage by means of texture analysis. Kidney 46:1721-7.

Morgan-Hughes GJ, Roobottom CA, Marshall AJ (2002). Aortic valve imaging with computed tomography: A review. Journal of Heart Valve Disease 11(5):604-11.

Muntzel M, Massy ZA, Ruellan N, Descamps-Latscha B, Lacour B, Drueke TB (2002). Chronic renal failure increases oxidative stress and accelerates atherosclerosis in apolipoprotein-E knock-out (EKO) mice (Abstract). Nephrol Dial Transplant 17:46.

Reinmuller R, Lipton MJ (1987). Detection of coronary artery calcification by computed tomography. Dynam Cardiovasc Imag 1:139-45.

Ser'on D, Moreso F, Gratin C, Vitría J, Colom E, Griny'o JM, Alsina J (1996). Automated Classification of Renal Interstitium and Tubules by Local Texture Analysis and a Neural Network. Anal Quant Cytol Histol 18:410-9.

Serra J (1982). Image Analysis and Mathematical Morphology. Vol I. London: Academic Press.

Serra J (1988). Image Analysis and Mathematical Morphology. Vol II: Theoretical Advances. London: Academic Press.

Serra J (1989). Toggle mappings. In: Simon JC, ed. From Pixels to Features. Amsterdam: North Holland, 61-72.

Soille P (1999). Morphological image analysis. Berlin, Heidelberg: Springer-Verlag.

Tanenbaum SR, Dondos GT, Veselik KE, Prendergast MR, Brundage BH, Chomka EV (1989). Detection of calcific deposits in coronary arteries by ultrafast computed tomography and correlation with angiography. Am J Cardiol 63:870-2.

Tomaževič D, Likar B, Pernuš F (2002). Comparative evaluation of retrospective shading correction methods. J Microsc-Oxford, 208:212-23.

Zhang SH, Reddick RL, Piedrahita JA, Maeda N (1992). Spontaneous hypercholesterolemia and arterial lesions in mice lacking apolipoprotein E. Science Oct 16;258(5081):468-71. 